NASA Technical Memorandum 101317

\title{
Comparison of the Bidirectional Reflectance Distribution Function of Various Surfaces
}

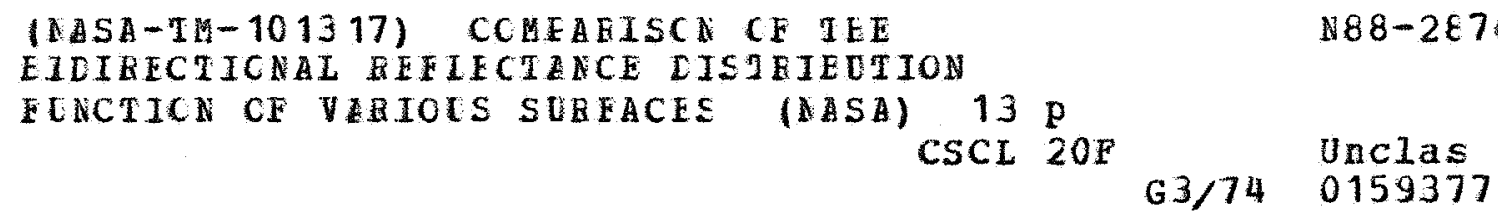

Rene Fernandez, Richard G. Seasholtz, and Lawrence G. Oberle Lewis Research Center Cleveland, Ohio

and

Jaikrishnan R. Kadambi

Case Western Reserve University

Cleveland, Ohio

Prepared for the

32nd Annual International Technical Symposium on Optical and Optoelectronic Applied Science and Engineering sponsored by the Society of Photo-Optical Instrumentation Engineers San Diego, California, August 14-19, 1988

\section{N/SA}


Comparison of the Bidirectional Reflectance Distribution

\title{
Function of Various Surfaces
}

Rene Fernandez, Richard G. Seasholtz, and Lawrence G. Oberle National Aeronautics and Space Administration

Lewis Research Center

Cleveland, Ohio 44135

and

Jaikrishnan R. Kadambi

Case Western Reserve University

Cleveland, Ohio 44106

\begin{abstract}
SUMMARY
This paper describes the development and use of a system to measure the Bidirectional Reflectance Distribution Function (BRDF) of various surfaces. The BRDF measurements will be used in the analysis and design of optical measurement systems, such as laser anemometers. An argon ion laser $(514 \mathrm{~nm})$ was the light source. Preliminary results are presented for eight samples: two glossy black paints, two flat black paints, black glass, sand blasted aluminum, unworked aluminum, and a white paint. $\mathrm{A} \mathrm{BaSO}_{4}$ white reflectance standard was used as the reference sample throughout the tests. The reflectance characteristics of these surfaces are compared in this paper.
\end{abstract}

\section{INTRODUCTION}

The geometrical reflectance properties of a surface is of importance in many fields and has a broad range of applications. Some examples are: minimizing stray light reflections for space Telescopes',? modeling the performance of solar energy collectors ${ }^{3}, 4$ and knowing how surface reflections affect the signal-to-noise (SNR) ratio in laser anemometry (IA) measurements ${ }^{5}$. The latter application is of relevance in this paper. The reflectance characteristics of surfaces are expressed in terms of the Bidirectional Reflectance Distribution Function (BRDF).

The results from this study can be used to define the surface model used in a computer simulation of an LA system used near a wall. Preliminary results are presented for eight samples: two glossy black paints, two flat black paints, black glass, sand blasted aluminum, unworked aluminum, and a white paint. With an accurate surface model, the optical system can be optimized ${ }^{6}$. Later LA experiments would be required to verify the performance predictions of the computer model.

Aluminum was used as the base material for all the paints because it is a commonly used structural material for fluid flow test facilities and models. Black Schott NG-1 neutral density filter glass was tested because it is frequently used in the construction of optical systems, such as light traps. 
Therefore the objective of this study was to measure the BRDF of various surfaces and to compare the results in order to pick candidates for the surface models.

\section{APPARATUS \& TEST METHODS}

The BRDF is a function that specifies and describes the geometrical reflectance properties of a surface. The BRDF is a function of four angles (two angles for the incident radiation and two angles for the reflected radiation), and the surface's reflecting properties ${ }^{7}$ :

$\operatorname{BRDF}\left(\theta_{j}, \phi_{i} ; \theta_{r}, \phi_{r}\right) \equiv d L_{r}\left(\theta_{i}, \phi_{i} ; \theta_{r}, \phi_{r} ; E_{i}\right) / d E_{i}\left(\theta_{i}, \phi_{j}\right)$

where: $\theta=$ polar angle

$\phi=$ azimuthal angle

$i=$ incident

$r=$ reflected

$\mathrm{L}=$ reflected radiance

$\mathrm{E}=$ incident irradiance

The angles are illustrated in figure 1. The surface's reflecting properties manifest themselves in the reflected radiance of the incident irradiance.

Figure 2 shows a schematic representation of the optical configuration. The reason for the circuitous beam path is that the same laser is used for two experiments, this BRDF study and LA studies. When the removable mirror is in place (as in figure 2), it intercepts the laser beam and reflects it back to the sample holder used in this study. Lenses LI and L2 are used to focus the laser beam on the sample surface. The laser beam was linearly polarized in the upward direction. Therefore, the incident beam on the sample surface was polarized perpendicularly to the measurement plane, i. e. it had "S" polarization.

The sample was mounted on a two-axis goniometer and the photodetector was mounted on an arm (Stage A) that could be rotated \pm $45^{\circ}$ as shown in figure 2. The goniometer could only rotate the sample holder about two orthogonal axes; stage $B$ rotated the sample holder in the horizontal plane and stage $C$ rotated it in the vertical plane. Because clockwise rotations, $i$. $e$. rotations about the normal to the sample surface, were not possible, $\phi_{\text {, }}$ and $\phi_{r}$ were combined into one difference angle, $\delta \phi$. Where $\delta \phi$ is the difference between the incident azimuthal angle, $\phi_{i}$, and the exitent azimuthal angle, $\phi_{r}$. Conversion equations were developed that related this angle as well as the polar angles, $\theta_{1}$ and $\theta_{r}$, to the actual experimental configuration angles, $i$. $e$. the goniometer angles and the rotational positioner angle. Because these equations were highly non-linear, a computer routine based on the Newton Raphson technique was used.

A Computer Numerical Control (CNC) positioning controller received these transformed angles from a PDP-11/44 computer and placed the rotational positioner stage (A stage) and goniometers ( $B$ and $C$ stages) 
at the correct angles. The photodetector was attached to stage $A$, $41.45 \pm 0.05 \mathrm{~cm}$ from the sample. The data recording was automated; the A stage would sweep in an arc on command from the computer. When the $A$ stage would stop after each angle increment, the light intensity striking the photodetector would be recorded on disk. The increment angle between data points was $2^{\circ}$ for all the data shown in this paper.

Table 1 lists the relevant features of the various components in the test apparatus, and Table 2 lists the samples tested. As shown in Table 1, the Argon ion laser $(514.5 \mathrm{~nm})$ was operated at output powers relevant to IA, approximately 1 watt. There were two exceptions to this power level. When the Chemglaze $\mathrm{z} 302$ sample was tested, scorching would occur for long exposures to any power level above 0.35 watts. Therefore, the chemglaze was tested at a power level of 0.3 watts. Scorching would occur at 0.6 watts for the sikkons 443-3-17 sample. Therefore, that sample was tested at a level of 0.51 watts. Drift in beam power was minimized in two ways. First, the laser was run in light control mode which uses a feedback circuit to maintain constant power output. Second, the data collection program constantly monitored the laser power. If the power varied by more than $\pm 2 \%$ an error message was issued.

Stray radiation was minimized by enclosing the test table in black velvet. A small hole was left for the incoming laser light. Background radiation runs were made with no samples and the laser set at various output power levels. For these, the laser beam did not strike the sample holder, but exited into the room through another small hole. These background light intensities were subtracted from the test sample light intensities for the corresponding laser power levels.

Table 2 lists the eight samples that were tested. The stock aluminum was tested at two different striation orientations. It was oriented with the striations horizontally and then it was tested again with the striations angled at $60^{\circ}$ from horizontal. The black glass sample was tested on two bases, a black glossy base and a white lambertian base. All the painted samples were prepared by depositing a layer of paint, from an airbrush, on the surface of a smooth aluminum base and setting aside to cure as per the manufacturers instructions.

The Schott NG-1 black glass was tested on two different bases, the glossy black Chemglaze $\mathrm{Z} 302$ and the white Baso 4 reference, in order to compare the subsurface scatter effects. Figure 3 illustrates the reflections from the upper and lower surfaces of a glass sample.

The reference sample method of computing BRDFs was used. The reference sample was a $\mathrm{BaSO}_{4}$ packed powder which was nearly an ideal white Lambertian reflector. The BRDF of a Lambertian surface is defined to be:

$$
\mathrm{BRDF}=\rho / \pi
$$

where: $p=$ the absolute reflectance of the surface 
The reflectance of the test sample, $p=0.991 \pm 0.005$ at $500 \mathrm{~nm}$, was taken from the manufacturer's specification sheet for our particular lot number.

Once the BRDF of a reference surface is known, the BRDF of a test sample can be found by ${ }^{8}$ :

$$
\mathrm{BRDF}_{\mathrm{s}}=\mathrm{BRDF}_{\mathrm{ref}}\left(\mathrm{V}_{\mathrm{s}} / \mathrm{V}_{\text {ref }}\right)
$$

where: $V_{\text {ref }}=$ the reference sample's photodetector voltage

$\mathrm{V}_{\mathbf{s}}=$ the test sample's photodetector voltage

After the data recording was completed, another computer program would take the light intensites that were recorded on the computer disk and plot the BRDF profiles. It computed the BRDF by using the system parameters, i. e. Iaser power, gain settings, and angular positions, that were stored on disk at the start of each test run.

\section{DISCUSSION OF RESULTS}

Plots which show the BRDF for the test samples are presented in figures 4 to 9. The BRDF is presented in the figures by a polar plot. The radial scale shows the BRDF level while the circumferential scale shows the angular position of the photodetector (figure 2). Data were recorded for a photodetector angle sweep from $+45^{\circ}$ to $-45^{\circ}$. Some of the plots show an angle less than $45^{\circ}$ on one side of the normal to the surface and an angle greater than $45^{\circ}$ on the other side. This was due to stage $B$ rotating the sample to achieve the desired beam incidence angle. The angle of incidence is indicated in the figure by the arrow labeled $I$. Because the scale was automatically selected by the computer plotting package, scale changes must be considered when comparing one figure to another.

Note that the sharp drop off, in the direction of the incident beam, observed in some of the graphs, is because the photodetector was behind mirror M2 (figure 2). When the photodetector is behind this mirror, it is in a $3^{\circ}$ eclipse region. All data shown are for "inplane" measurements: the surface normal, the incident beam, and the scattered beam lie in a common plane. Three laser beam incident angles, $1^{\circ}, 10^{\circ}$, and $20^{\circ}$, are presented in the figures.

Interesting observations were made. Figure 4 shows results for the two flat black paints, Sheffield Pot-belly black and 3M ECP-2200 on the same graph for easy comparison. For all the incident angles, the pot belly black had a higher BRDF than the 3M ECP-2200, although both were flat blacks. Since both the paints were deposited on the same type of substrate, the differences can be attributed to the paints' properties.

Similarly, the two glossy blacks (figures 5 and 6 ) differed in their BRDF functions; the sikkons 443-3-17 sample had a consistently larger maximum BRDF, $i$. e. the specular scatter, than the Chemglaze Z302 sample. The diffuse scatter portion of the distributions, however, had the opposite relationship. The Chemglaze $\mathrm{z} 302$ had a higher BRDF than the sikkons 443-3-17 sample. For these figures, only 
the diffuse scatter portion of the BRDF is shown. The dashed lines indicate that the large BRDF values corresponding to the specular reflections are off scale. The spread out shape of the distribution indicates that cratering or surface damage of the Chemglaze $\mathrm{Z302}$ had occurred. Upon visual inspection of the surface, small indentations were observed. Although scorching did not occur, a laser power level of 0.3 watts is still too high to avoid surface damage.

The aluminum samples demonstrated the affect of various surface finishes on the same type of material. As shown in figure 7 the sand blasted sample had a much smoother, diffuse, reflectance function, while the unworked samples exhibited specular behavior. Rotating the unworked sample by $60^{\circ}$ drastically cut down the BRDF values and made the reflection less specular.

The white Nextel sample was close to the lambertian reference, as figure 8 illustrates. For higher incidence angles (figure 8c), however, the the lambertian behavior breaks down and the sample reflects light back towards the incident beam path more than it reflects the light in the specular direction. The Nextel surface would be desirable for LA applications where a uniform BRDF is desirable.

Figure 9 shows the behavior of the schott NG-1 black glass sample. As with the figures for the glossy black paints, the larger BRDF values corresponding to specular scatter are off scale. The diffuse scattering from the black glass was significantly less than the diffuse scattering from the black paints. This surface had the lowest diffuse scatter characteristics of all the samples tested.

The data for the black glass with the two different base surfaces, the Chemglaze $\mathrm{Z302}$ and the white $\mathrm{BaSO}_{4}$ reference, showed that the BRDF values were similar for both bases. This indicates that not much light was getting through the black glass and onto the base surface. Therefore subsurface scatter effects were not very significant.

Data for out-of-plane reflectance properties were obtained during the research effort. Although these data are not presented in this paper, the results were predictable. The diffuse scattering samples, Nextel 101-A10, Sheffield Pot Belly Black, and 3M ECP-2200, had distributed BRDF profiles. The more specular scattering samples had peaked BRDF profiles. Furthermore, the test apparatus is being used to evaluate the reflectance properties of new samples.

\section{SUMMARY OF RESULTS}

of the samples evaluated, the schott NG-1 black glass sample had the best overall characteristics: high specular scatter for the one reflection angle, low diffuse scatter for other angles, and not sensitive to high incident power levels. Although the Chemglaze $\mathrm{Z} 302$ and the Sikkons 443-3-17 satisfied the first two requirements they are both censitive to incident light levels.

The Nextel 101-A10 would be the desirable sample for applications that require a predictable and uniform BRDF profile. An example of this is are IAA studies were the photodiode may be aimed along the 
specular reflection angle and there is a chance that it may be overwhelmed by reflections from a bright surface. A lambertian surface would minimize this possibility.

Using this highly automated BRDF measurement system a database of candidate surfaces for LA measurements near a wall has been established. Furthermore, there is little data in the literature on BRDF measurements made with the high incident light levels that were used in this study. We have shown that although a material may have good reflectance properties, an important consideration is the incident light intensity. Too high an intensity may damage the material. This set of data should be useful for researchers needing reflectance data for laser based optical measurement systems.

\section{ACKNOWLEDGMENTS}

We thank Mr. Gordon Driver for his quick repair of any electrical problems encountered and $\mathrm{Mr}$. Jerry Nelson for his help with mechanical problems.

\section{REFERENCES}

1. D. B. Griner, "Bidirectional Reflectance Distribution Function (BRDF) measurements of stray light suppression coatings for the space Telescope (ST)," in Space Optics, SPIE Vol. 183, (1979).

2. S. M. Smith, "BRDF measurements of sunshield and baffle materials for the IRAS telescope," NASA TM-84235, Ames Research Center, Calif., (June 1982).

3. R. B. Pettit and B. L. Butler, "Laser ray trace and Bidirectional reflectometry measurements of various solar concentrators," in Conf.-770953-4, U. S. Department of Energy, Concentrating Solar Collector Conf., Altanta (Sep 1977).

4. A. A. De Silva and B. W. Jones, "Bidirectional spectral reflectance and directional-hemispherical spectral reflectance of six materials used as absorbers of solar energy," in Solar Energy Materials, Vol. 15, pp. 391-401, North-Holland, Amsterdam (1987).

5. A. Mishina, N. S. Vlachos, and J. H. Whitelaw, "Effect of wall scattering on SNR in laser doppler velocimetry", Applied Optics, Vol. 18 , No. 14, pp. 2480-2485 (1979)

6. R. G. Seasholtz, L. G. Oberle, and D. H. Weikle, "Optimization of Fringe-Type Laser Anemometers for Turbine Engine Component Testing", AIAA-84-1459 (1984)

7. F. E. Nicodemus, J. C. Richmond, and J. J. Hsia, Geometrical considerations and nomenclature for reflectance, National Bureau of Standards, Washington, D. C., (Oct. 1977) .

8. F. O. Bartell, E. L. Dereniak, and W. L. Wolfe, "The theory and measurement of Bidirectional Reflectance Distribution Function (BRDF) and Bidirectional Transmittance Distribution Function (BTDF)", in Radiation Scattering in Optical systems, SPIE Vol. 257, (1980). 


\section{Table 1. BRDF System Component Summary}

Laser:

Sample spot size: Collecting Area:

Solid angle: Light power meter: Signal amplifier:

A stage:

B \& C stages:
5 watt Argon ion (514.5 nm) model, light control operation, linearly polarized

$2.5 \mathrm{~mm}(0.0984 \mathrm{in})$ diameter

$0.1781 \mathrm{~cm}^{2}\left(0.0276 \mathrm{in}^{2}\right)$

$1.037 \times 10-4$ steradians

P.I.N. Si Photodiode, $\pm 5 \%$ accuracy analog output Variable gain amplifier, $10 \mathrm{~Hz}$ bandwidth Arm mounted on a rotational positioner, $\pm 45^{\circ}$ Goniometers, $\pm 45^{\circ}$

Table 2. Test Samples

Sample

Aluminum, sand blasted

Aluminum, stock

Black glass (Schott NG-1)

Chemglaze $\mathrm{Z} 302$

Nextel 101-A10

Sheffield Pot-belly black

Sikkons 443-3-17

$3 \mathrm{M}$ ECP-2200
Fabrication

Stock aluminum, was sand blasted stock aluminum with striations $1 \mathrm{~mm}$ thick, glossy black glass Black paint sprayed on aluminum White paint sprayed on aluminum Flat black sprayed on aluminum Black paint sprayed on aluminum Flat black sprayed on aluminum

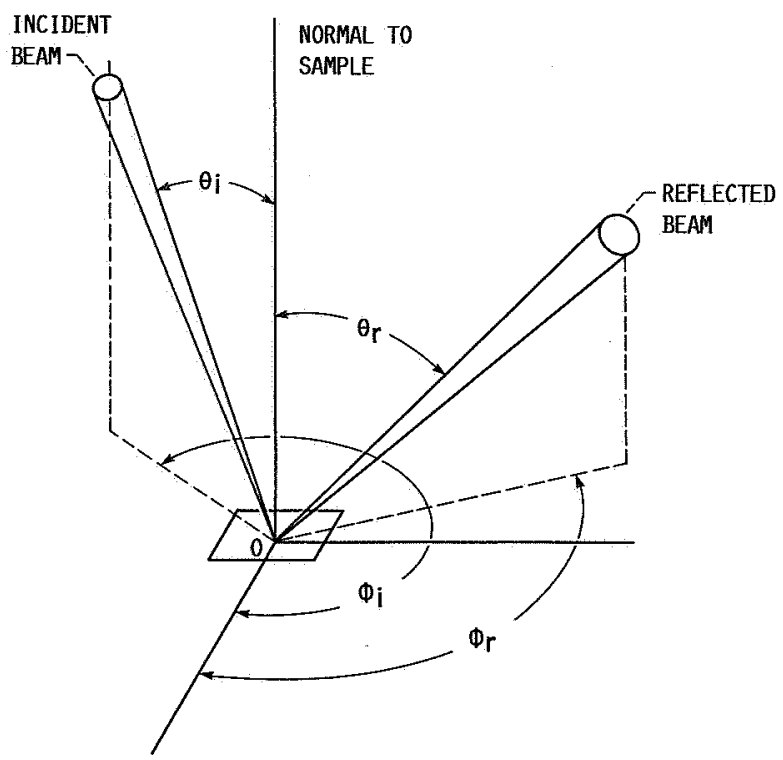

FIGURE 1. - ANGLES FOR THE INCIDENT AND REFLECTED BEAMS. 


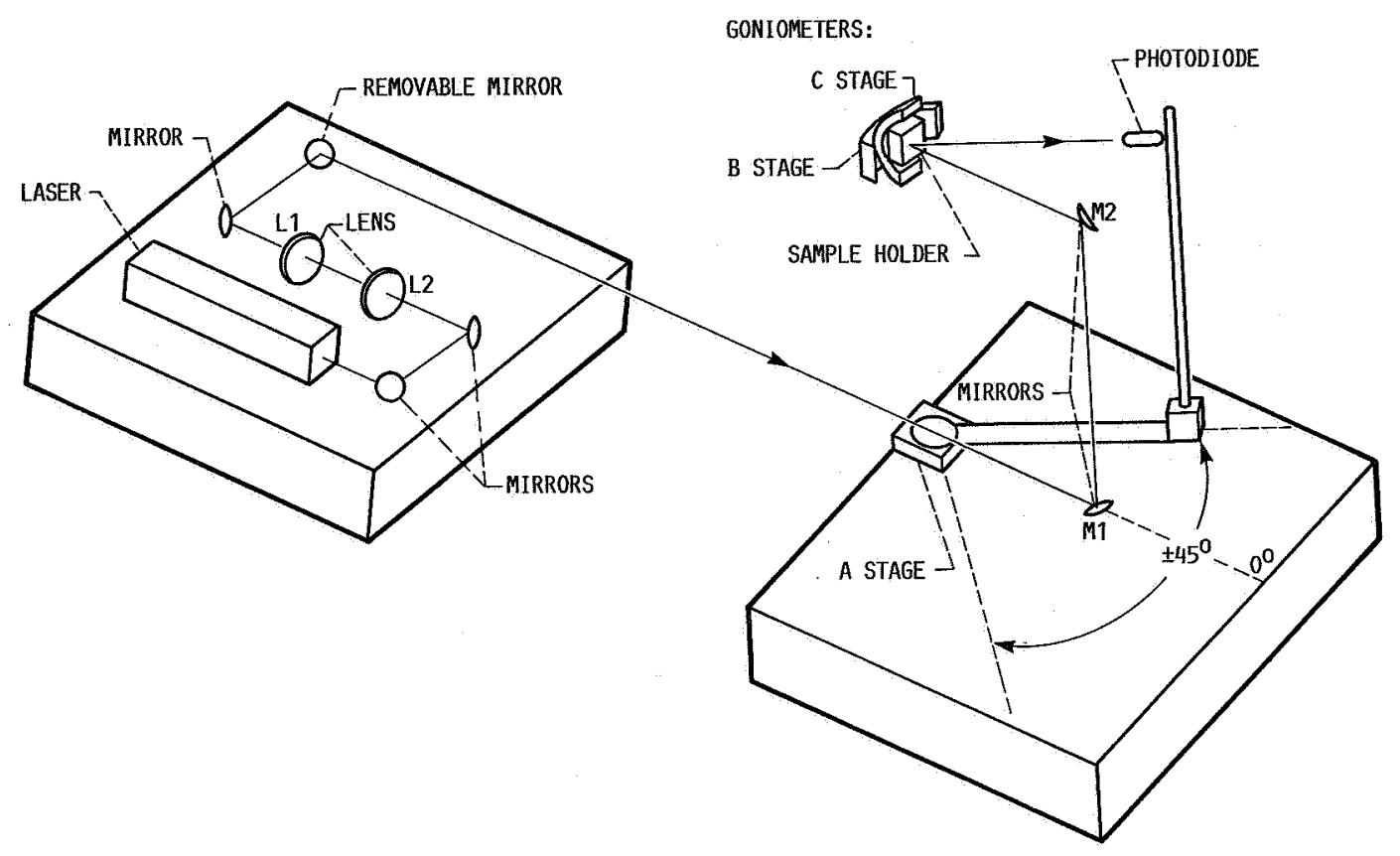

FIGURE 2. - THE OPTICAL CONFIGURATION.

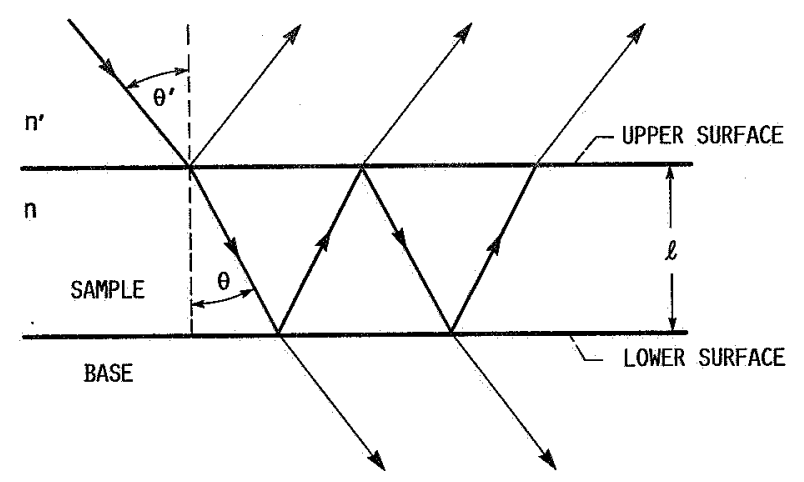

FIGURE 3. - SUBSURFACE SCATTER EFFECT. 


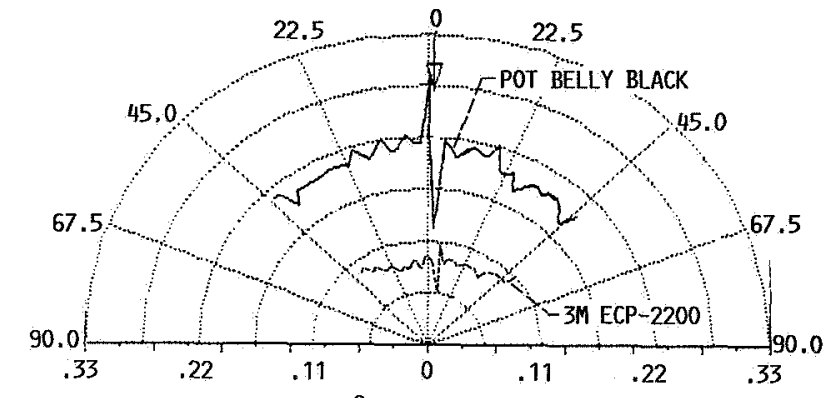

(a) $1^{0}$ INCIDENT ANGLE.

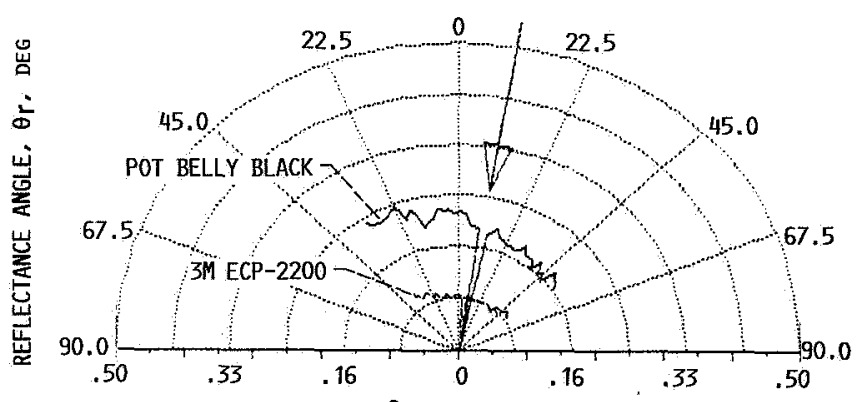

(b) $10^{\circ}$ INCIDENT ANGLE.

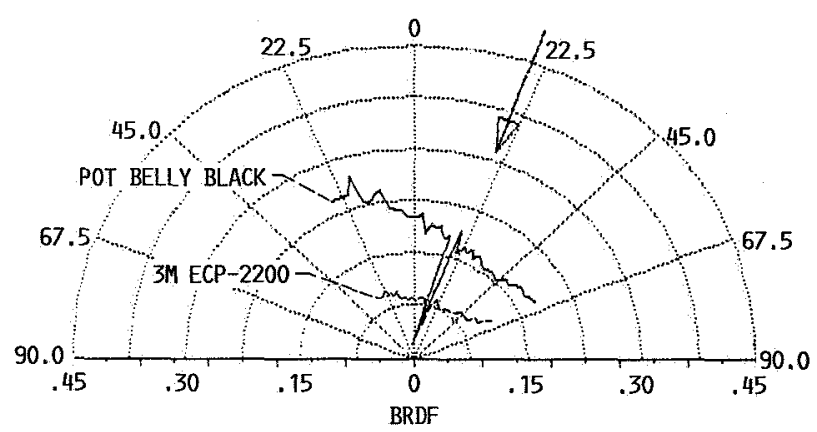

(c) $20^{\circ}$ INCIDENT ANGLE.

FIGURE 4. - POLAR PLOT OF BRDF FOR 3M ECP-2200 VERSUS SHEFFIELD POT BELLY BLACK.

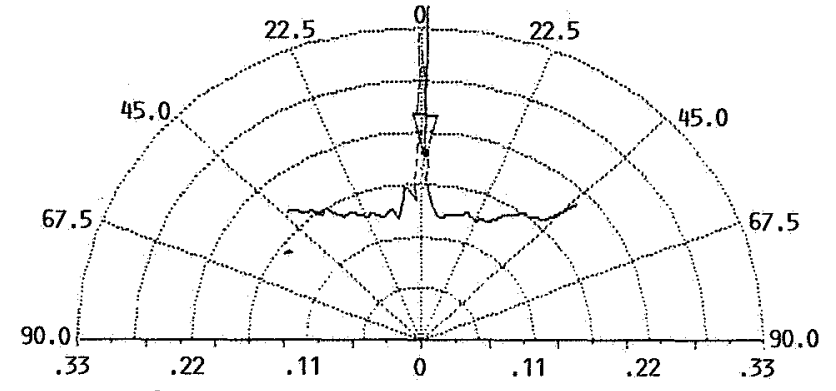

(a) $1^{\circ}$ INCIDENT ANGLE. MAXIMUM BRDF OF 5.79 AT $0^{\circ}$ IS OFF SCALE.

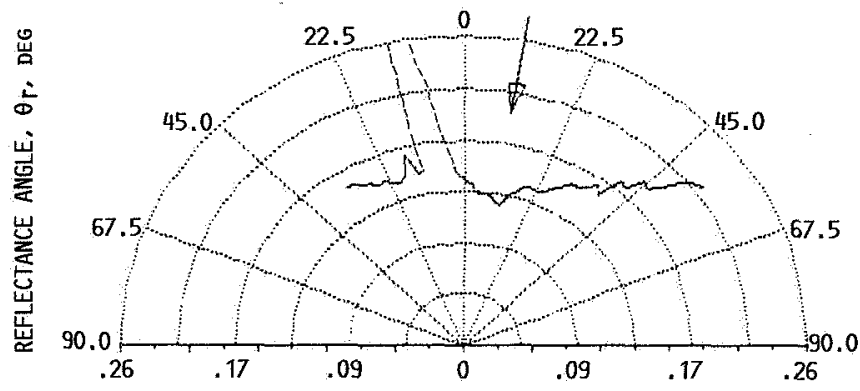

(b) $10^{\circ}$ INCIDENT ANGLE. MAXIMUM BRDF OF 7.18 AT $-10^{\circ}$ IS OFF SCALE.

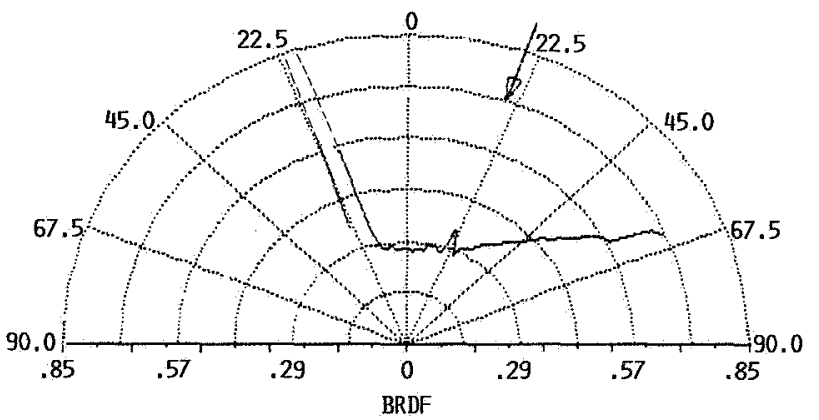

(c) $20^{\circ}$ INCIDENT ANGLE. MAXIMUM BRDF OF 10.38 AT $-20^{\circ}$ IS OFF SCALE.

FIGURE 5. - POLAR PLOT OF BRDF FOR CHEMGLAZE Z302 CHEMGLAZE Z302 (SURFACE DAMAGED). 


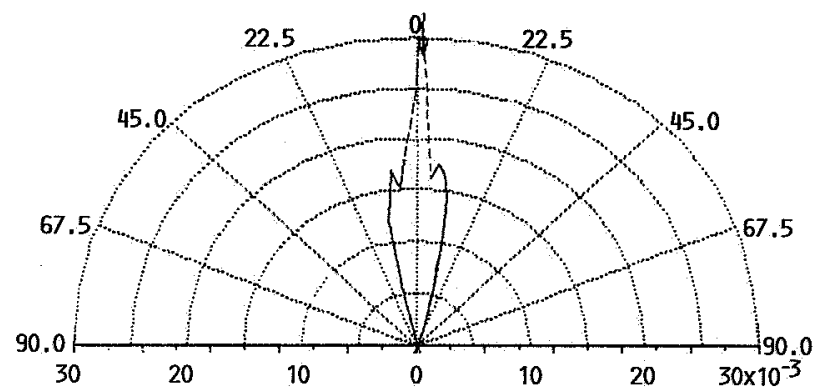

(a) $1^{0}$ INCIDENT ANGLE. THE MAXIMUM BRDF OF 22.84 AT $0^{\circ}$ IS OFF SCALE.

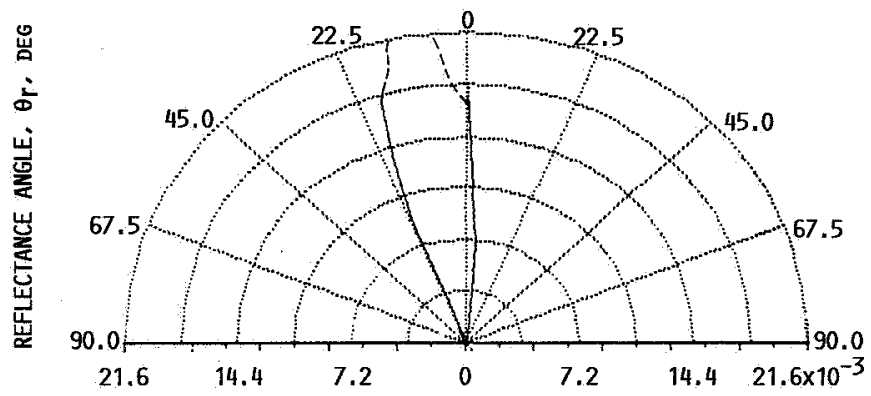

(b) $10^{\circ}$ INCIDENT ANGLE. THE MAXIMUM BRDF OF 11.90 AT $-10^{0}$ IS OFF SCALE.

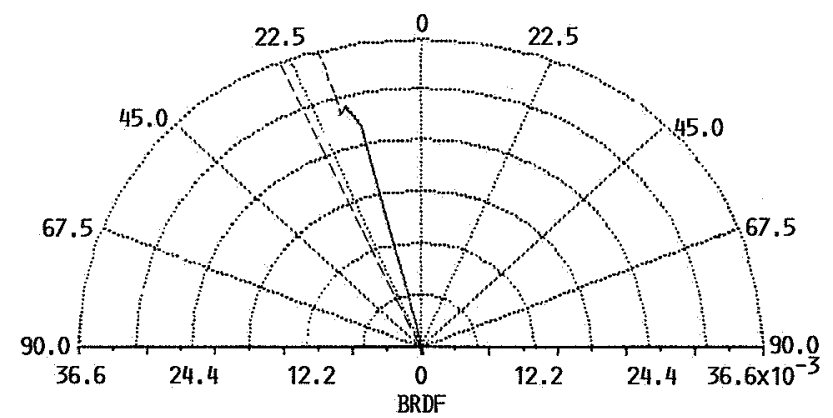

(c) $20^{\circ}$ INCIDENT ANGLE. THE MAXIMUM BRDF OF 17.65 AT $-20^{\circ}$ IS OFF SCALE.

FIGURE 6. - POLAR PLOT OF BRDF FOR SIKKONS 443-3-17.

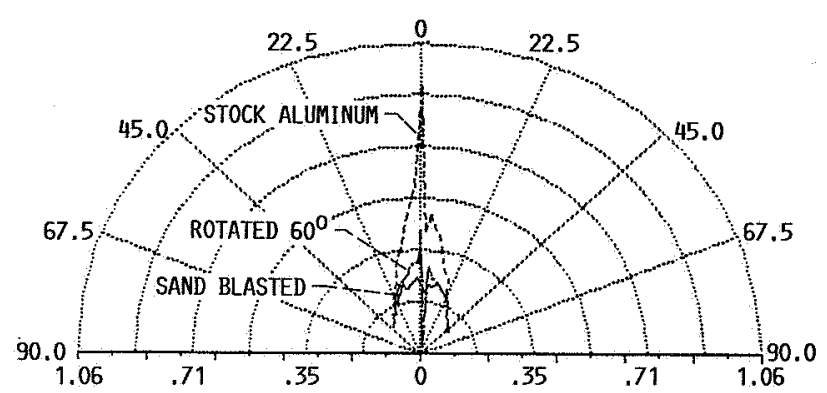

(a) $1^{0}$ INCIDENT ANGLE.
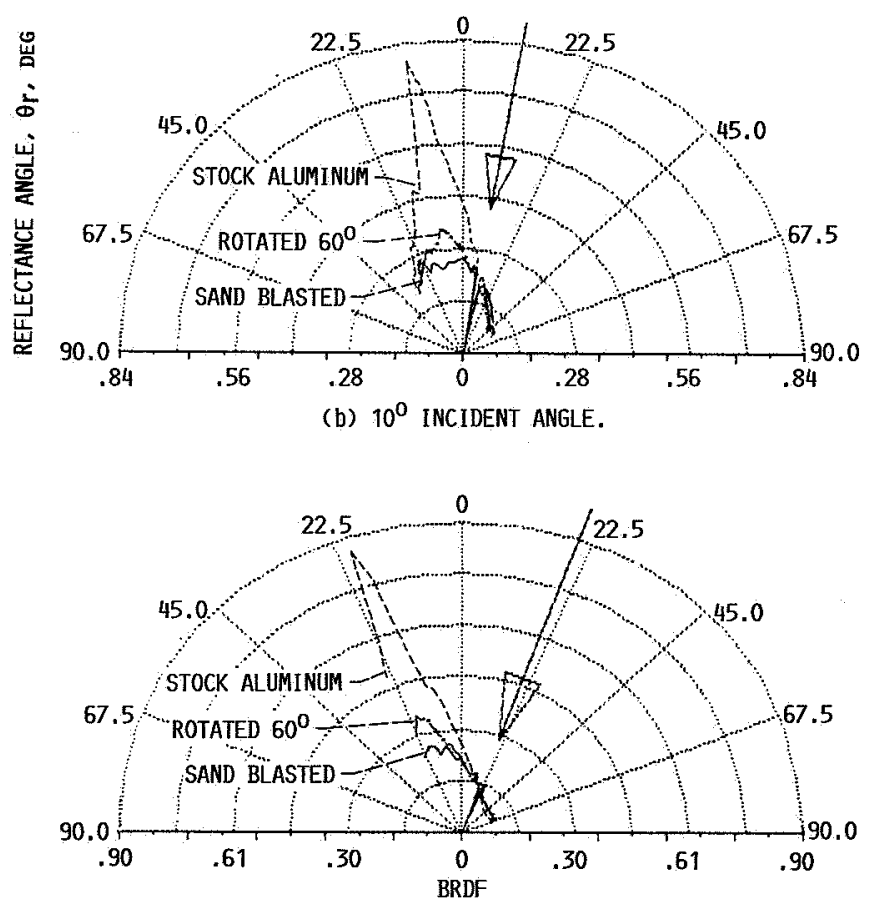

(c) $20^{\circ}$ INCIDENT ANGLE.

FIGURE 7. - POLAR PLOT OF BRDF FOR THE ALUMINUM SAMPLES. 


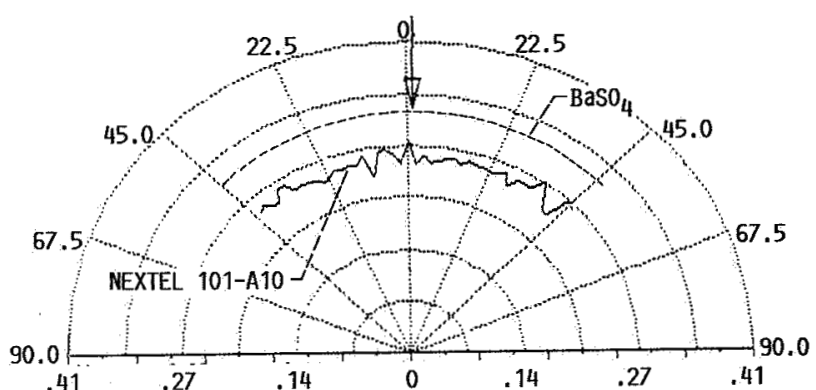

(a) $1^{\circ}$ INCIDENT ANGLE,

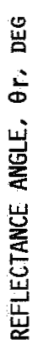

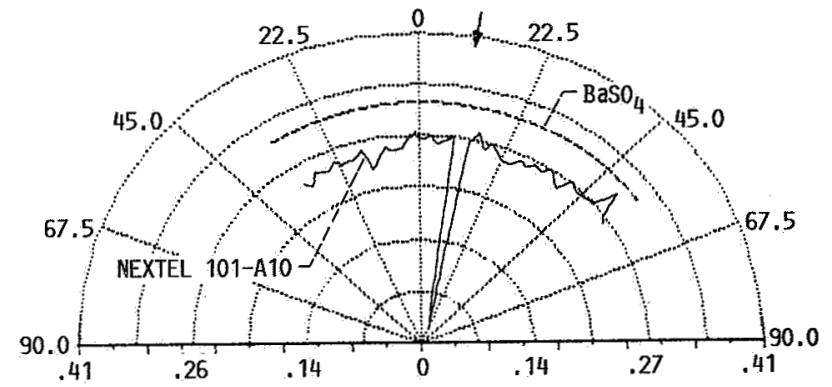

(b) $10^{\circ}$ INCIDENT ANGLE.

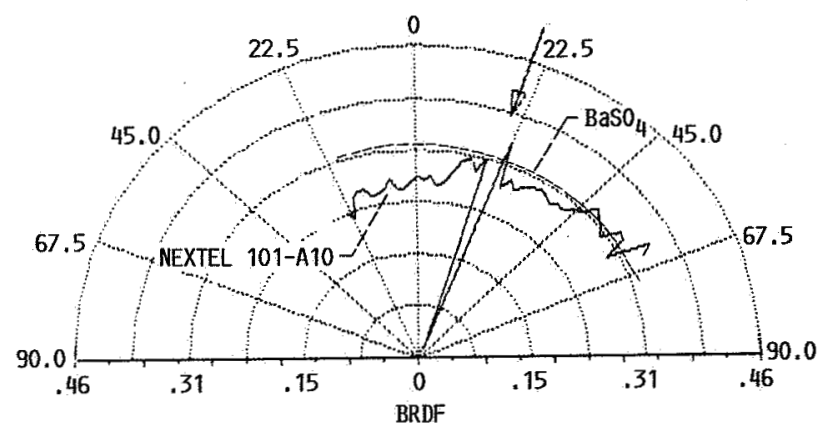

(c) $20^{\circ}$ INCIDENT ANGLE.

FIGURE 8. - POLAR PLOT OF BRDF FOR NEXTEL 101-A10 VERSUS $\mathrm{BaSO}_{4}$.

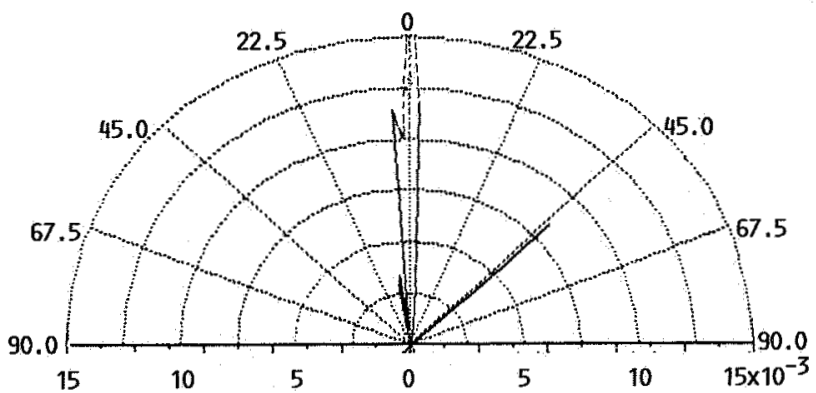

(a) $1^{0}$ INCIDENT ANGLE. THE MAXIMUM BRDF OF 427 AT $0^{\circ}$ IS OFF SCALE.

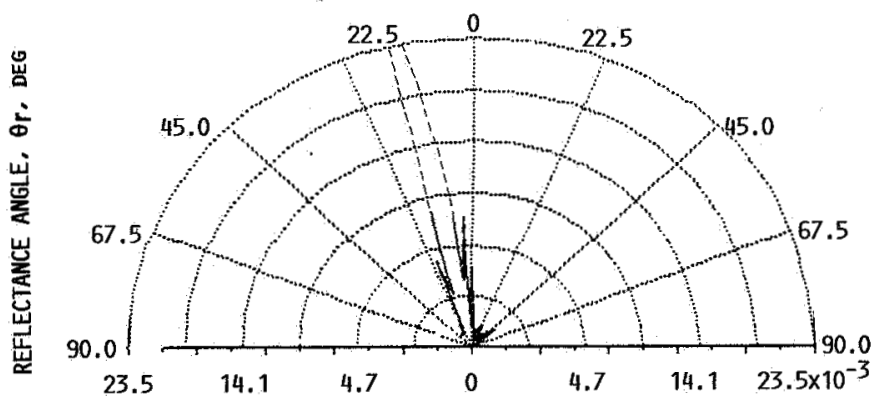

(b) $10^{\circ}$ INCIDENT ANGLE, THE MAXIMUM BRDF OF 1.15 AT $-10^{\circ}$ IS OFF SCALE.

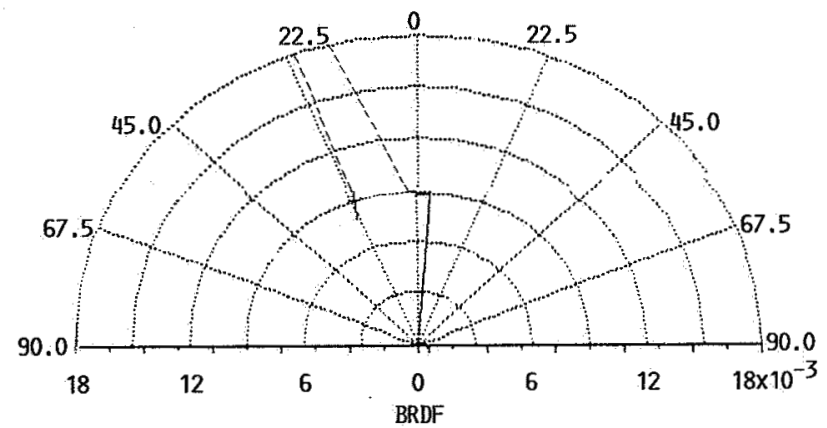

(c) $20^{\circ}$ INCIDENT ANGLE. THE MAXIMUM BRDF OF 14.79 AT $-20^{\circ}$ IS OFF SCALE.

FIGURE 9. - POLAR PLOT OF BRDF FOR BLACK GLASS. 


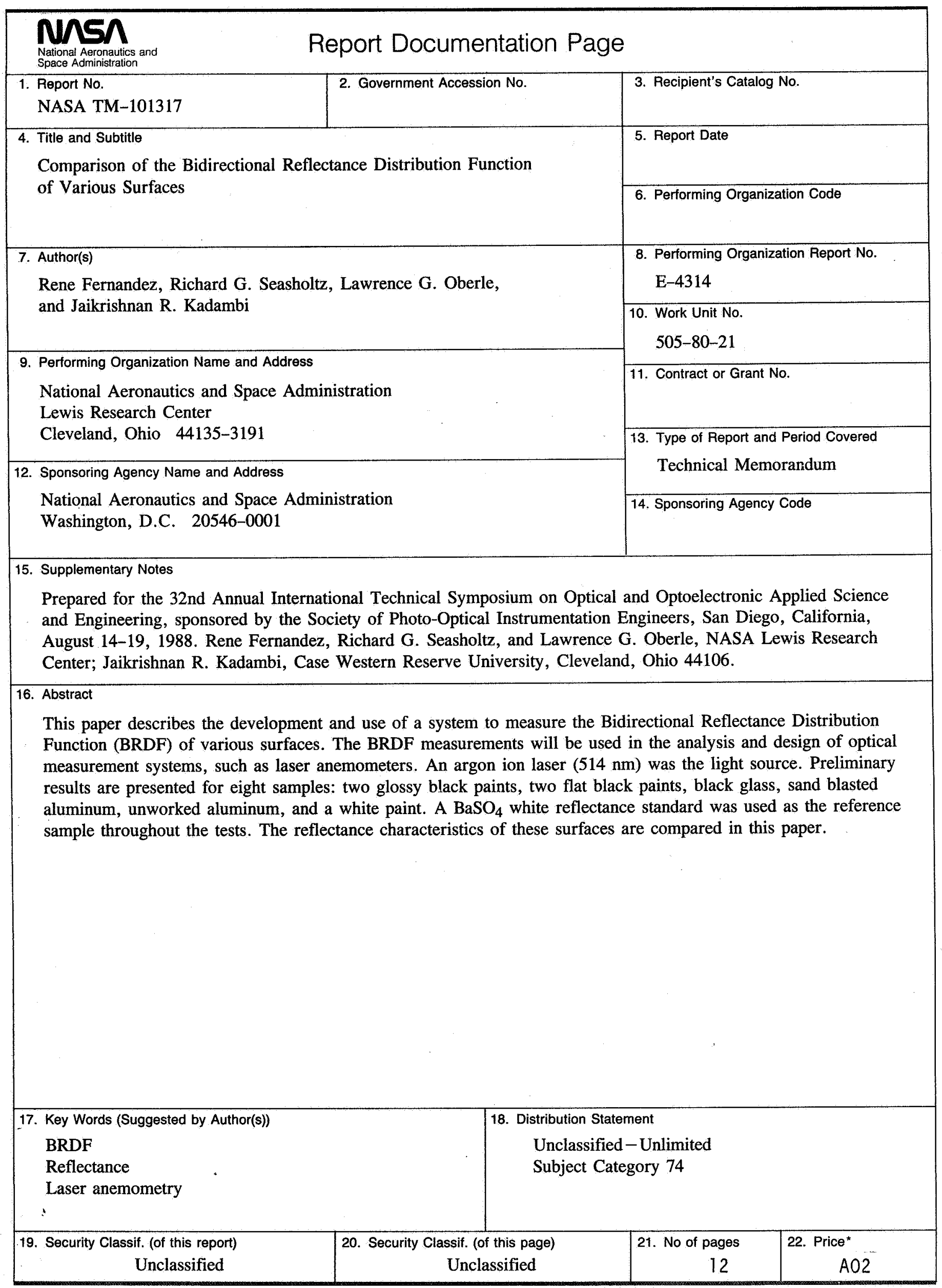

